\title{
No Reservations Required: Achieving Fairness between Wi-Fi and NR-U with Self-Deferral Only
}

\author{
Ilenia Tinnirello* \\ University of Palermo, Consorzio Nazionale \\ Interuniversitario per le Telecomunicazioni (CNIT) \\ ilenia.tinnirello@unipa.it \\ Szymon Szott ${ }^{*}$ \\ AGH University \\ szott@agh.edu.pl
}

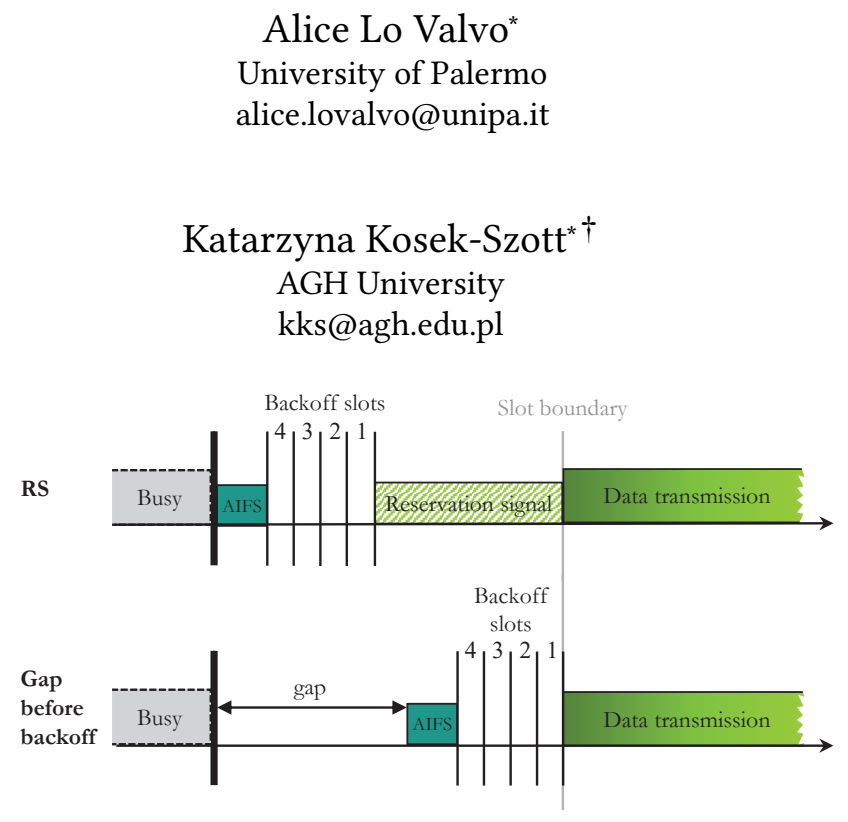

Figure 1: Two approaches to aligning an LBT procedure with the slot boundary of a scheduled access network such as NR-U: reservation signal (top) and gap (bottom).

Conference on Modeling, Analysis and Simulation of Wireless and Mobile Systems (MSWiM '21), November 22-26, 2021, Alicante, Spain. ACM, New York, NY, USA, 11 pages. https://doi.org/10.1145/3479239.3485680

\section{INTRODUCTION}

There is growing research interest in solving the problem of coexistence in unlicensed (shared) bands between wireless networks based on diverse paradigms: random access (local and personal area networks: Wi-Fi, Bluetooth) and scheduled access (cellular networks: Long Term Evolution License Assisted Access, LTE LAA; and New Radio-Unlicensed, NR-U). In this paper, we consider Wi-Fi and NR-U as examples of technologies adopting the random and scheduled access approaches, respectively. Coexistence between $\mathrm{Wi}-\mathrm{Fi}$ and NR-U is based on having both technologies use a listen before talk (LBT) procedure [34], which is considered fairer in comparison to duty cycling used by LTE-U [31]. However, LBT was designed for random channel access whereas scheduled systems require that their transmissions start at the beginning of a slot boundary [14]. Synchronizing this boundary with the end of LBT requires that either (a) channel access is initialized after a selfdeferral (i.e., gap period) which is not interrupted by some other transmission or (b) the NR-U node transmits a reservation signal (RS) which blocks the channel until the start of the slot boundary (Figure 1).

The RS-based approach is often used in coexistence analysis and was shown to preserve fairness (Section 2) but it is not explicitly 
mentioned in 3GPP specifications [3]. Furthermore, it may lead to a waste of radio resources ${ }^{1}$ and, therefore, it has been criticized not only by IEEE [23] but also by researchers [8, 14, 17]. Furthermore, ETSI's Broadband Radio Access Networks (BRAN) committee is currently working on an updated version of the LBT specification which may disallow the use of RS [21]. On the other hand, as shown in [13], the gap mechanism, in its present form, does not always preserve fairness in coexistence between scheduled and randomaccess systems, especially for large synchronization slots (especially for the lengths allowed for NR-U in the $5 \mathrm{GHz}$ band: from 0.25 to 1 $\mathrm{ms})$.

Therefore, in this work we propose to discard the RS-based approach, treat the gap mechanism as a partial backoff during LBT procedure, and use contention window $(\mathrm{CW})$ settings adjusted to the number of coexisting nodes (Section 4). This not only ensures $\mathrm{Wi}-\mathrm{Fi} / \mathrm{NR}-\mathrm{U}$ coexistence fairness but also avoids wasting radio channel resources and improves aggregate Wi-Fi/NR-U throughput. We validate our idea for several topology settings. Then, we show by extensive simulations that the proposed approach outperforms the RS-based channel access and provides significant throughput and fairness gains. Additionally, we develop an analytical model (Section 3.2) which can be used either to replace long-lasting simulations or for $\mathrm{CW}$ tuning (i.e., finding the optimal Wi-Fi/NR-U CW settings) using the descendent gradient method. Finally, we implement a long short-term memory-based (LSTM) regression model to predict $\mathrm{Wi}-\mathrm{Fi} / \mathrm{NR}-\mathrm{U} \mathrm{CW}$ settings resulting in $\mathrm{Wi}-\mathrm{Fi} / \mathrm{NR}-\mathrm{U}$ coexistence fairness (Section 5). This last approach could also be used in practice, in which the simulation samples could be replaced by real data samples.

\section{STATE OF THE ART}

The coexistence between random (Wi-Fi) and scheduled-based (LAA, NR-U) access in shared channels has been intensely studied in recent years [33]. So far, most works have focused on Wi-Fi/LAA coexistence and assumed that LAA uses RSs [7, 11, 18, 26].

In a recent trend, researchers have started to propose augmenting cellular networks with machine learning techniques to improve channel allocations, transmission scheduling, and coexistence fairness. Typically, they propose changes to the standard LTE-U/LTELAA operation or provide new ways of monitoring Wi-Fi conditions and traffic demands $[4,5,19,29]$.

Other papers attempt to modify the standard LBT scheme. Saadat et al. suggest tuning the $\mathrm{CW}$ and TXOP parameters based on observed network load to improve overall performance [27]. In terms of maintaining coexistence, instead of using a RS, they have LAA eNBs send a CTS-to-self to inform Wi-Fi nodes of their impending transmissions. Han et al. [10] also propose to intelligently tune CWs for both LAA and Wi-Fi nodes using reinforcement learning. However, they assume that LAA nodes start transmitting immediately after LBT. Ali et al. also tune CW parameters, but using neural networks [5]. They assume RS-based channel access but also consider issues related to collisions and updating the $\mathrm{CW}$, including the impact of delayed CW update due to LTE's HARQ.

${ }^{1}$ For $1 \mathrm{~ms}$ synchronization slots only 1 bit is transmitted per $0.5 \mathrm{~ms}$ (on average) prior to each data transmission.
Huang et al. [12] address the impact of hidden and exposed nodes on $\mathrm{Wi}-\mathrm{Fi} / \mathrm{LAA}$ coexistence. They propose a receiver-initiated transmission mode for LAA (based on a design for mmWave networks [15]), which is able to reduce the collision rate and improve throughput. However, following [15], they do not consider the necessity of LAA transmissions to be synchronized to slot boundaries. For mmWave bands, this requirement can be lifted, especially if the slot length is short. However, this is not the case for the $5 \mathrm{GHz}$ band. Additionally, Saleem et al. [28] suggest completely avoiding inter-network collisions by having Wi-Fi and LAA transmit in duty cycles. The authors define the optimization problem and solve it analytically. Again, synchronization slot boundaries are not considered. Furthermore, Loginov et al. suggest an alternative LBT implementation, which does use RSs, but in a new way, in the form of short busy tones [16]. This provides collision detection and resolution which leads to increased network performance, at the cost of changes in LAA/NR-U channel access behavior.

Further in the topic of coexistence between NR-U and Wi-Fi, [22] discusses issues in the $6 \mathrm{GHz}$ band, while [25, 32] provide coexistence studies for the mmWave $(60 \mathrm{GHz})$ band. In particular, [22] is a tutorial paper, in which challenges and opportunities for the next generation Wi-Fi and 5G NR-U in the $6 \mathrm{GHz}$ bands are described. Additionally, [25] presents a simulation model developed for ns-3, which takes into account both LBT and duty cycle (LTEU-like) channel access modes for NR-U. Furthermore, [32] finds stochastic models for SINR and data rate under the assumption of downlink transmissions.

In summary, most of the literature either does not consider slot boundaries or assumes the use of RS-based channel access. Our goal is to find a solution which would take into consideration the characteristics of NR-U transmissions but without resorting to reservation signals.

\section{MODELLING CHANNEL ACCESS}

In this section we briefly present the LBT channel access rules and how they are implemented by Wi-Fi and NR-U. Next, we develop analytical and simulation models of $\mathrm{Wi}-\mathrm{Fi} / \mathrm{NR}-\mathrm{U}$ coexistence.

\subsection{Channel Access Rules}

Channel access for both Wi-Fi and NR-U follow the LBT procedure defined in ETSI specification EN 301893 [2]. Before transmitting, nodes are required to perform LBT by observing the channel to be idle for $16 \mu \mathrm{s}$, referred to as the short inter-frame space (SIFS) in Wi-Fi. Afterwards, contention is resolved by having nodes wait for a fixed and random amount of $9 \mu$ s backoff slots. By default (i.e., for best effort traffic), there are three fixed slots (referred to as the arbitration inter-frame space number, AIFSN in Wi-Fi) and a random number of backoff slots chosen between zero and the current $\mathrm{CW}$ value, which is set to CWmin after each successful transmission and doubled (up to CWmax) before each retransmission.

The IEEE 802.11-2016 [1] (Wi-Fi) channel access rules closely follow these LBT specifications. Furthermore, since Wi-Fi requires in-band signalling to confirm unicast transmissions, each successful transmission is followed by an immediate acknowledgement (ACK) frame transmission. 
3GPP Rel-16 [3] (NR-U) also follows the LBT rules, where the above-mentioned exponential backoff mechanism is used for data (Cat-4) transmissions. However, NR-U differs from Wi-Fi in two important aspects. First, acknowledgements are sent over the licensed band $^{2}$. Second, NR-U uses slot-based scheduling: transmissions are organized into radio frames, each composed of 10 sub-frames (slots) and NR-U transmissions can start only at slot boundaries. This requires alignment between the LBT procedure and slot boundary, which can be resolved using a gap period or an reservation signal (RS) transmission. The primary focus of this study is improving fairness when NR-U uses gap-based channel access, which allows avoiding unnecessary channel occupation by the RSs. The gap can be placed before or after the backoff countdown [13]; we assume the former approach as in [20].

While NR-U slots are by default $1 \mathrm{~ms}$ long, NR-U allows for flexible slot lengths, by increasing the subcarrier spacing. For the $5 \mathrm{GHz}$ bands, the slot lengths can be configured to $250 \mu$ s or $500 \mu \mathrm{s}$. In addition to this slot-based scheduling, NR-U introduces the concept of mini-slots for non-slot-based scheduling [13], but we do not consider these aspects in this paper.

\subsection{Analytical Model}

The main assumptions of our model are the following. First, all devices are within hearability range (i.e., there are no hidden nodes). This means that the end of a data transmission resets the channel state for all nodes (i.e., they all simultaneously begin to count the AIFS time, etc.). Second, the network operates under saturation conditions (i.e., a full-buffer traffic model in all nodes), which means that transmissions occur consecutively on the channel. Third, there are only downlink transmissions, i.e., initiated by Wi-Fi access points (APs) or NR-U base stations (next generation NodeBs, gNBs). Under these assumptions, the channel activity can be described in consecutive contention rounds, including an idle time required for solving the contention, as well as a transmission time, in which the winner station holds the channel (as shown in Figure 2).

We propose to model the behavior of each contending node $k$ by deriving the steady-state distribution $B_{k}($.$) of the random process$ $b_{k}($.$) which describes the evolution of the backoff counter value$ at the end of each transmission/collision event delimiting the contention round. Each discrete time $n=1,2, \ldots$ identifies the start of the $n-t h$ contention round, in which the node with the shortest backoff expiration time gains the right to perform a transmission attempt. Such a backoff expiration time depends simply on the backoff counter for Wi-Fi nodes, while for NR-U nodes it also depends on the additional gap interval. On the basis of previous studies [13], the gap interval can be considered as a random variable, uniformly distributed between 0 and the maximum synchronization interval $\Delta$. For simplicity, we assume that all nodes use a constant (but adjustable) $\mathrm{CW}$, to better clarify the impact of the random gap interval on the contention process. Generalizations to exponential backoff mechanisms are straightforward. To some extent, the contention process between $\mathrm{Wi}-\mathrm{Fi}$ and NR-U nodes is similar to the contention process between two access categories (ACs) of the enhanced distributed channel access (EDCA) defined in the IEEE

${ }^{2} \mathrm{NR}-\mathrm{U}$, like LAA, is based on carrier aggregation with devices having a connection in both licensed and unlicensed bands [34].

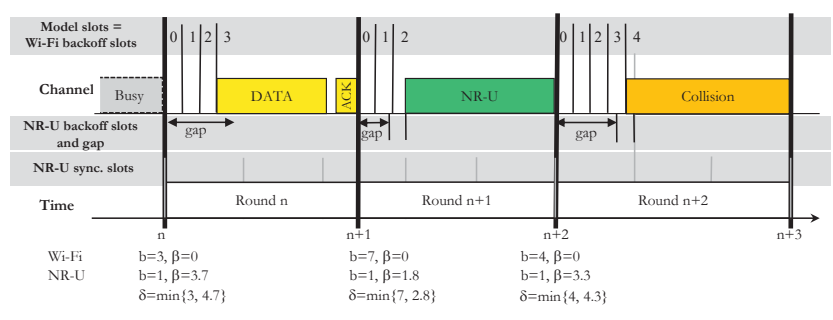

Figure 2: An example of the backoff evolution process in a coexistence scenario with one $\mathrm{Wi}-\mathrm{Fi}$ and one NR-U node. The channel is divided into contention rounds, which in turn include an idle contention time, indexed in consecutive backoff slots $b$, and a final channel holding time (i.e., the time required to transmit a Wi-Fi DATA and ACK frame or a series of NR-U subframes). Note that the model slots are enumerated in ascending order, whereas the Wi-Fi backoff slots are usually numbered in descending order.

802.11 standard, in which the arbitration inter-frame space (AIFS) ${ }^{3}$ value used by one service class (corresponding to NR-U nodes) is a random variable (equivalent to gap interval $+A I F S_{\mathrm{BE}}$ interval for the best effort (BE) traffic) rather than a fixed interval.

To model Wi-Fi/NR-U coexistence, we use an approach similar to the one proposed in [30]. Let $N$ be the total number of contending nodes, with $N_{W}$ Wi-Fi nodes and $N_{N}=N-N_{W}$ NR-U nodes. Let $\beta_{k}$ the additional gap interval (measured in backoff slots) required for each $k$-th contenting node, the value of which is set to zero for Wi-Fi nodes.

The idle time required for starting a new transmission on the channel in the current contention, expressed in the number of backoff slots, is equal to:

$$
\delta(n)=\min _{j=1,2, \ldots N}\left[b_{j}(n)+\beta_{j}(n)\right] .
$$

Note that channel access times for NR-U nodes are not in general synchronized with channel access times for Wi-Fi nodes, because the gap interval is not necessarily an integer number of backoff slots. Let $C S$ be the carrier sensing time (which we assume equal to one half backoff slots) and $\xi$ be the nodes whose backoff expiration time is in the interval $\delta(n)+C S$, i.e., the nodes starting a new transmission attempt in the current contention. The backoff evolution law can be defined as:

$$
b_{k}(n+1)=\left\{\begin{array}{l}
b_{k}(n)-\lceil\delta(n)\rceil, k \notin \xi \\
\quad \text { for Wi-Fi, } \\
b_{k}(n)-\max \left\{\left\lceil\delta(n)-\beta_{k}(n)\right\rceil, 0\right\}, k \notin \xi \\
\quad \text { for NR-U, } \\
\operatorname{rand}\left(C W_{k}\right), k \in \xi,
\end{array}\right.
$$

where $\operatorname{rand}\left(C W_{k}\right)$ is an integer extracted randomly in the range $\left[0, C W_{k}\right]$, where $C W_{k}$ is the CW used by node $k$.

Figure 2 shows an example of the backoff evolution process of one Wi-Fi and one NR-U node in three consecutive contention rounds. In the first round, the Wi-Fi node expires first its backoff

${ }^{3}$ For each $\mathrm{AC}, A I F S_{\mathrm{AC}}$ is calculated as: $A I F S N_{\mathrm{AC}}$ times the backoff slot length plus SIFS. 
counter equal to 3 slots, while the NR-U node keeps its backoff counter frozen (where the gap is longer than the backoff expiration time). A new random backoff counter equal to 7 is extracted for the next round. In the second round, the NR-U node has a smaller access time and wins the contention, thanks to a new gap value equal to 1.8 backoff slots. Since the NR-U transmission is performed after two idle slots from the last transmission, the Wi-Fi backoff counter is decremented by three units. Finally, in the last contention round the two nodes collide, despite the fact that their access times do not coincide exactly. This is due to the required channel sensing time $C S$.

3.2.1 Per-node Backoff Process. Consider now the evolution of the backoff process of a tagged Wi-Fi node $w$ at the end of a contention. We sequentially index the idle backoff slots that follow our model time as slot $0,1, \ldots, M$, as well as the channel holding time concluding each contention, as shown in Figure 2. The number of backoff idle slots in each contention is bounded by the minimum value among the maximum contention time of each node, i.e., $M=$ $\min \left\{C W_{w}, C W_{n}+\lceil\Delta\rceil-1\right\}$. Assume that we know the probability $T_{w}(i)$ that any other node starts its first transmission attempt in the $i$-th slot. We remark that each transmission attempt is not in general synchronized to the start of a new backoff slot, because of the gap interval employed by NR-U nodes. For example, in the last contention round illustrated in Figure 2, the NR-U node starts its transmission after 4.3 idle slots from the end of the previous channel holding time (i.e., within the slot numbered as 4 , but not exactly at the start of such a slot).

For Wi-Fi nodes, we can characterize the steady-state backoff probability $B_{w}(j)$ to have a backoff counter equal to $j$ as follows:

$$
\begin{aligned}
& B_{w}(j)=\sum_{r=1}^{C W_{w}-j} B_{w}(j+r) \cdot T_{w}(r-1)+ \\
& +\frac{1}{C W_{w}} \cdot \sum_{r=0}^{C W_{w}} B_{w}(r) \cdot\left[1-\sum_{l=0}^{r-1} T_{w}(l)\right] .
\end{aligned}
$$

At the end of a new contention round, the backoff counter of the tagged node $w$ may be equal to $j$ for two different reasons:

- it has been decremented to $j$ starting from a greater value $j+r$ (with $r \in\left\{1,2, \ldots, C W_{w}-j\right\}$ ), because a transmission attempt has been performed by other nodes after $r-1$ idle slots;

- it has been randomly set to a new value equal to $j$ right after a transmission attempt performed by the tagged node.

Note that the expiration of a generic backoff counter equal to $r$ occurs when no other node transmits in the consecutive slots numbered from 0 to $r-1$. This happens with probability $1-\sum_{l=0}^{r-1} T_{w}(l)$

For NR-U nodes, at each contention round, the backoff process is resumed only after the expiration of the gap interval. Therefore, assuming we know the $T_{n}(i)$ probability that a tagged NR-U node $n$ sees a transmission performed by other nodes within the $i$-th slot, the backoff counter is updated only when $i$ is greater than $\beta_{n}$. Let $G(i)$ be the probability that the random gap is in the interval between $i$ and $i+1$ backoff slots, with $i \in\{0,1, \ldots,\lceil\Delta\rceil-1\}$. In other words, we approximate such a probability with a uniform discrete distribution, whose generic value $G(i)$ is equal to $1 /\lceil\Delta\rceil$.
Let $Z_{n}(r)$ be the probability distribution of the difference between the number of backoff slots in the current contention round and the gap. Such a probability can be expressed as the convolution between $T_{n}($.$) and G($.$) . With abuse of notation, we extend the defi-$ nition of $T_{n}(i)$ by setting $T_{n}(i)=0$ for $i<0$. We can then express $Z_{n}(r)$ as $\sum_{l=0}^{\lceil\Delta\rceil-1} T_{n}(r+l) /\lceil\Delta\rceil$, with $r \in\left\{-\lceil\Delta\rceil+1, \ldots, C W_{n}\right\}$. The backoff evolution process can be characterized as:

$$
\begin{array}{r}
B_{n}(j)=B_{n}(j) \cdot \sum_{r=-\lceil\Delta\rceil}^{0} Z_{n}(r-1)+\sum_{r=1}^{C W_{n}-j} B_{n}(j+r) \cdot Z_{n}(r-1)+ \\
+\frac{1}{C W_{n}} \cdot \sum_{r=0}^{C W_{n}} B_{n}(r) \cdot\left[1-\sum_{l=0}^{r-1} Z_{n}(l)\right]
\end{array}
$$

At the end of each contention round, the backoff counter of the tagged NR-U node $n$ may be equal to $j$ for three different reasons:

- it is equal to the previous contention value, because other nodes transmit before the gap expiration and the backoff process is not resumed;

- it has been decremented to $j$ starting from a greater value $j+r$ because a transmission attempt was performed by other nodes after $r$ slots from the gap expiration;

- it has been randomly set to a value equal to $j$ after the backoff counter of the tagged node was resumed and decremented to zero.

3.2.2 Contention Idle Time. The medium access process can be characterized as a function of the backoff probability distributions of the contending nodes. In case all the nodes belonging to the same technology employ the same constant $\mathrm{CW}$ value, they will result in the same backoff distribution $\left(B_{w}(\right.$.$\left.) or B_{n}().\right)$.

In each contention round, the probability $Q(i)$ to observe at least $i$ idle slots is given by the probability that all Wi-Fi nodes have a backoff counter greater than $i$, and all NR-U nodes have the sum of the gap interval and backoff counter greater than $i$. With abuse of notation, let $B_{n}(l)=0$ for $l<0$, and let $E_{n}(r)=$ $\sum_{l=0}^{\lceil\Delta\rceil-1} B_{n}(r-l) /\lceil\Delta\rceil$ be the probability that the sum between the gap interval and the NR-U backoff counter expires within slot $r$. It follows that

$$
Q(i)=\left(\sum_{j=i+1}^{C W_{w}} B_{w}(j)\right)^{N_{W}} \cdot\left(\sum_{j=i+1}^{C W_{w}} E_{n}(j)\right)^{N_{N}}
$$

The probability $Q_{h}(i)$ that a generic node $h$ observes at least $i$ idle slots in a contention round can be obtained similarly to the previous expression, but removing the tagged node from the set of contending nodes:

$$
Q_{h}(i)=\left(\sum_{j=i+1}^{C W_{w}} B_{w}(j)\right)^{N_{W}-(h==w)} \cdot\left(\sum_{j=i+1}^{C W_{w}} E_{n}(j)\right)^{N_{N}-(h==n)}
$$

where $(h==w)$ and $(h==n)$ are Boolean values, which are equal to 1 when the node uses, respectively, Wi-Fi or NR-U technology. We can now express the probability $T_{h}(i)$ to have a transmission within the $i$-th backoff slot as:

$$
T_{h}(i)=Q_{h}(i)-Q_{h}(i+1)
$$


The system of equations (3), (4), (6) and (7) define $B_{w}($.$) and B_{n}($. as a function of $T_{h}($.$) , and T_{h}($.$) as a function of B_{w}($.$) and B_{n}($.$) . It$ can then be solved iteratively by means of fixed point iterations. More specifically, the solution is obtained as follows.

Starting from an initial assumption on $B_{n}($.$) and B_{w}($.$) distribu-$ tions, we can readily compute the quantities in (6) and (7). These quantities are in turn used to update the backoff distributions according to (3) and (4). The backoff distributions are normalized before computing again the idle time distributions $Q_{n}($.$) and Q_{w}($.$) ,$ as well as the aggregated behavior of other transmission attempts $T_{h}($.). This cycle is repeated until the maximum difference between the probability distributions found in two different iterations is lower than a convergence threshold (set to 0.001 in our implementation).

3.2.3 Performance Figures. We focus on the derivation of the probability $P S_{w}\left(\right.$ or $P S_{n}$ ) that a Wi-Fi (or NR-U) node successfully transmits in a contention round. We are particularly interested to the analysis of the channel shares obtained by each technology; moreover, the absolute throughput experienced by each node employing a generic technology $h=w, n$ can be easily expressed as a function of its success probability $\left(P S_{h}\right)$, as discussed next. A transmission attempt is successful if a tagged node transmits during the $r$-th slot inside the contention round, while all other nodes are scheduled to transmit after the occurrence of such a slot. Therefore, we can express:

$$
\begin{gathered}
P S_{w}=\sum_{r=0}^{C W_{w}} B_{w}(r) Q_{w}(r+1), \\
P S_{n}=\sum_{r=0}^{C W_{n}+\lceil\Delta\rceil-1} E_{n}(r) Q_{n}(r+1) .
\end{gathered}
$$

We can also derive the average number $E[x]$ of idle backoff slots in each contention as:

$$
E[x]=\sum_{i=0}^{\min \left\{C W_{h}, C W_{n}+\lceil\Delta\rceil-1\right\}} i \cdot[Q(i)-Q(i+1)]
$$

The throughput of a node employing technology $h$ is then immediately achievable as:

$$
S_{h}=\frac{P S_{h} \cdot L}{E[x] \cdot \sigma+T_{t x}},
$$

where $T_{t x}$ is the channel holding time in each transmission attempt, which we assume to be the same for both Wi-Fi and NR-U nodes ${ }^{4}$ and $L$ is the frame size. The normalized airtime $A_{h}$ can be easily derived by replacing in the previous expression $L$ with the time interval spent for transmitting the frame payload.

\subsection{Simulation Model}

Since available network simulators lack an implementation of the gap-based approach, we developed our own Monte Carlo simulator of the channel access described in Section 3.1. We use the simulator to validate the correctness of the analytical model described in

\footnotetext{
${ }^{4}$ Generalizations to heterogeneous channel holding times are straightforward, by differentiating the probability to have a successful transmission performed by WiFi or NR-U node, as well as a collision involving homogeneous and heterogeneous technologies.
}

Table 1: Default simulation parameters

\begin{tabular}{ll}
\hline Parameter & Value \\
\hline Operation band & $5 \mathrm{GHz}$ \\
Wi-Fi CWmin, CWmax & 15,63 \\
NR-U CWmin, CWmax & 15,63 \\
Wi-Fi transmision duration (w/o ACK) & $2 \mathrm{~ms}$ \\
NR-U transmision duration & $2 \mathrm{~ms}$ \\
NR-U synchronization slot duration & $1000 \mu \mathrm{s}$ \\
\hline
\end{tabular}

Section 3.2 and to generate training data for the regression model described in Section 5. The correctness of the simulator was verified by experiments (cf. Section 4.4).

Following the same assumptions as for the analytical model (no hidden nodes, network saturation, downlink traffic), we design the simulator to iterate over consecutive contention rounds (Figure 2). Each contention rounds begins after a transmission on the channel completes and consists of:

- a waiting period - needed to resolve the contention, it comprises AIFS, backoff, and gap periods.

- a transmission period (channel holding time) - the node or nodes which won the contention in the waiting period begin their transmissions.

The duration of the contention rounds is variable, depending on the chosen backoff, data transmission duration, etc. For Wi-Fi, the transmission period excludes the time required for transmitting an acknowledgement (ACK) frame (if a single node won the contention). For NR-U, we assume out-of-band signalling, i.e., that ACKs are transmitted on the licensed channel.

The simulator logic determines which nodes win the contention by calculating the Wi-Fi nodes that have the lowest backoff value and the NR-U nodes that have the lowest backoff and gap value. Next, if the set of nodes which are determined to have won the contention consists of a single node, we consider this a successful transmission. Otherwise, there is a collision. Furthermore, the simulator implements the channel access rules described in Section 3.1 including channel access parameters, backoff countdown, CW doubling, etc. Table 1 provides the default simulation parameters (the RS method is straightforward and has no configurable parameters). Once a sufficiently large number $\left(10^{5}\right)$ of contention rounds have elapsed, the simulation ends. The $95 \%$ confidence intervals were too small to be displayed.

We measure the following performance metrics:

- normalized airtime $A$ - the total channel occupancy time (including ACKs for Wi-Fi and RS for NR-U, if used) related to successful transmissions of either technologies, normalized to the total simulation time,

- fairness - measured using Jain's fairness index calculated either over the normalized airtime of each node (simulation model) or over the per-technology average per-node normalized airtime (analytical model),

- joint airtime-fairness $F$ - our proposed metric which takes into account both high channel utilization as well as fairness in channel access, calculated as the product of the aggregate normalized airtime of all nodes and the fairness. 


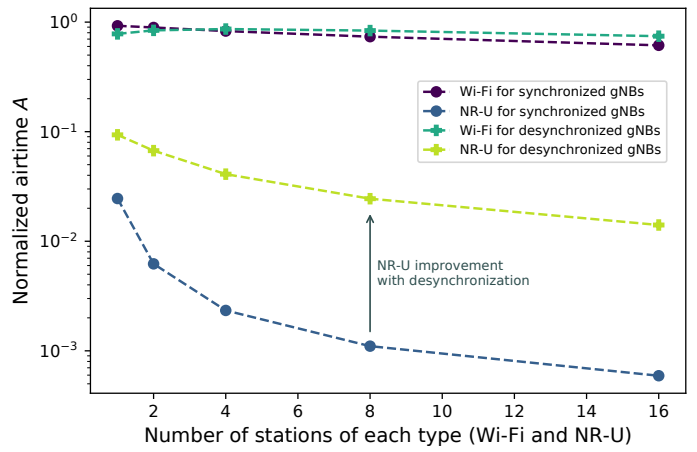

Figure 3: Simulation results of the impact of gNB synchronization on NR-U performance under coexistence with $\mathrm{Wi}$ Fi.

\section{OBSERVATIONS REGARDING IMPROVING NR-U PERFORMANCE}

Using the analytical model of Section 3.2 and the simulation model of Section 3.3, we investigate the performance of NR-U and Wi-Fi in a coexistence scenario (configured as described in Table 1) by answering several research questions in the following subsections.

\subsection{NR-U Synchronization}

The first question we posit is: should NR-U nodes (the gNBs) remain synchronized when operating on a common shared channel? If all contending gNBs are synchronized in the time domain, e.g., through GPS, then their synchronization slot boundaries start at the same time. Since the gNBs do not use a reservation signal, they will initiate a transmission exactly at the start of their slot boundary. Thus, they are destined to collide. This is corroborated by the results in Figure 3, where we compare fully synchronized gNBs with desynchronized gNBs (i.e., with randomly offset synchronization slots). The results lead us to formulate the following:

OBSERVATION 1. NR-UgNBs contending in shared channels should be desynchronized.

\subsection{Backoff Settings of NR-U gNBs}

If gNBs are descynchronized (i.e., their slot boundaries start at different times), their transmissions will not be initiated simultaneously. Also, they will experience different gap periods in each contention. These two factors render the backoff countdown of LBT unnecessary, as it no longer serves its purpose when the synchronization slot duration is large $(1000 \mu \mathrm{s})$. We tested this hypothesis by disabling backoff for $\mathrm{gNBs}$ (setting $\mathrm{CWmin}=\mathrm{CWmax}=0$ ). Our results (Figure 4) confirm that this leads to another improvement in terms of NR-U airtime share.

OBSERVATION 2. Backoff can be disabled for gap-based channel access if the synchronization slot duration is sufficiently large.

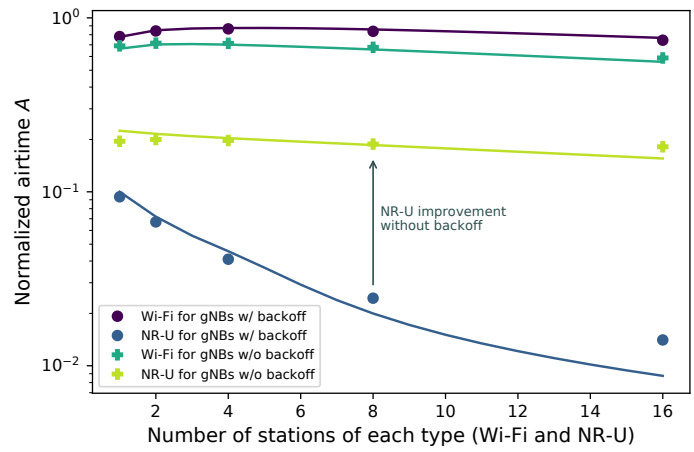

Figure 4: Impact of disabling backoff at gNBs on NR-U performance under coexistence with $\mathrm{Wi}$-Fi. Solid lines represent analytical model results, points - simulation results.

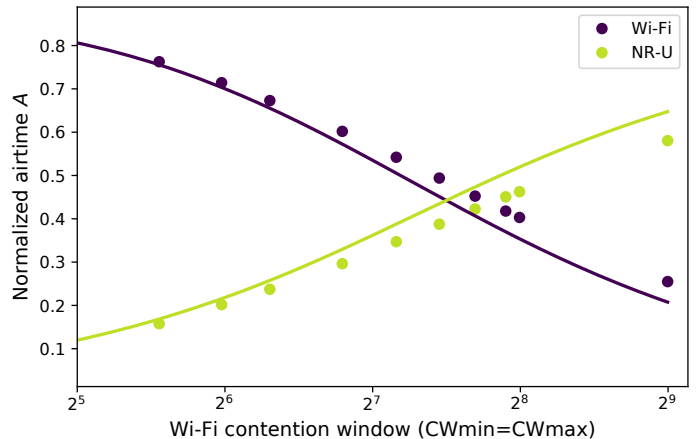

Figure 5: Example of achieving equal airtime distribution between $\mathrm{Wi}-\mathrm{Fi}$ and $\mathrm{NR}-\mathrm{U}$ by equalizing the delay in channel access for $N_{W}=N_{N}=2$ (assuming a fixed $\left.C W_{N}=0\right)$. Solid lines represent analytical model results, points - simulation results.

\subsection{Equalizing Per-Technology Airtime}

Observations 1 and 2 (desynchronizing gNBs and disabling backoff, respectively) have so far provided an improvement in NR-U airtime, but it is still an order of magnitude lower than that of Wi-Fi. This is because both technologies have unequal delay in accessing the channel: (a) Wi-Fi nodes wait for AIFS and the backoff time, (b) NR-U nodes wait for AIFS and a gap time distributed between 0 and the synchronization slot duration, and (c) backoff times are much shorter for Wi-Fi than gap periods for NR-U under the standard parameter set. Furthermore, it is well known that under saturation, appropriately selected constant CWs (adjusted to the number of coexisting nodes) can improve performance by avoiding unnecessary collisions [6]. The analytical and simulation results presented in Figure 5 provide an example supporting the following:

OBSERVATION 3. It is possible to achieve a fair airtime distribution between Wi-Fi and NR-U by finding optimal CW settings for both technologies. 


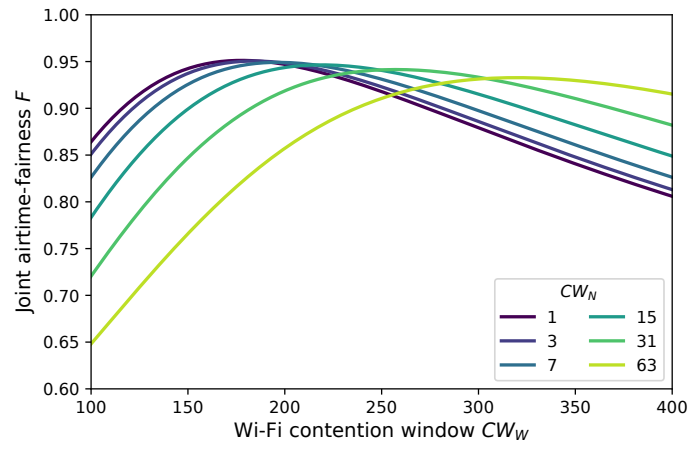

Figure 6: Maximizing joint-airtime fairness $F$ with different $\left(C W_{W}, C W_{N}\right)$ pairs for $N_{W}=N_{N}=2$ : several pair values lead to similar performance.

The optimal CW settings can be found using the analytical model of Section 3.2, which can compute the probability to win a contention round and the airtime of each contending node as a function of the contention parameters employed by the coexisting $\mathrm{Wi}$-Fi and NR-U technologies, the synchronization slot of NR-U nodes, and the number of contending nodes belonging to each technology. Let $f\left(C W_{W}, C W_{N}, \Delta, N_{W}, N_{N}\right)$ be the model function, whose outputs are $P S_{W}, A_{W}, P S_{N}$ and $A_{N}$. Although the function cannot be inverted in a closed form, it is possible to apply a numerical inversion method for adjusting the $\mathrm{CW}$ value of a node employing a given technology to achieve a desired airtime. For example, assuming the $C W_{N}$ value if fixed, it is possible to find the $C W_{W}$ value which leads to the equalization of airtimes for all the contending nodes. Starting from a given $C W_{W}(n)$ value at step $n$, considering the relative error on airtimes as a cost function to be set to zero, we can adjust the $\mathrm{CW}$ value of Wi-Fi nodes as:

$$
C W_{W}(n+1)=C W_{W}(n)-\left\lceil\frac{A_{N}(n)-A_{W}(n)}{A_{W}(n)} \cdot I\right\rceil,
$$

where $I$ is the iteration step (set in in our implementation to 64) Exemplary results of this type of $\mathrm{CW}$ tuning are shown in Figure 6 for $N_{W}=N_{N}=2$. Interestingly, the joint-airtime fairness $F$ is maximized for several $\mathrm{CW}$ pair values. Therefore, there are no universal CW settings.

Alternatively, simulation or analytical training data can be gathered and a regression model can be used to predict Wi-Fi/NR-U CW settings resulting in a high $F$, as later described in Section 5.

\subsection{Comparison with RS-based Channel Access}

Observations 1-3 have provided us with a configuration profile for Wi-Fi and NR-U, where the latter uses gap-based channel access. How does this profile compare with the case where gNBs use RSbased access? We use the joint airtime-fairness metric, introduced in Section 3.3, to compare the following channel access schemes for NR-U:

- reservation signal - gNBs use standard settings and an RS signal prior to transmission,

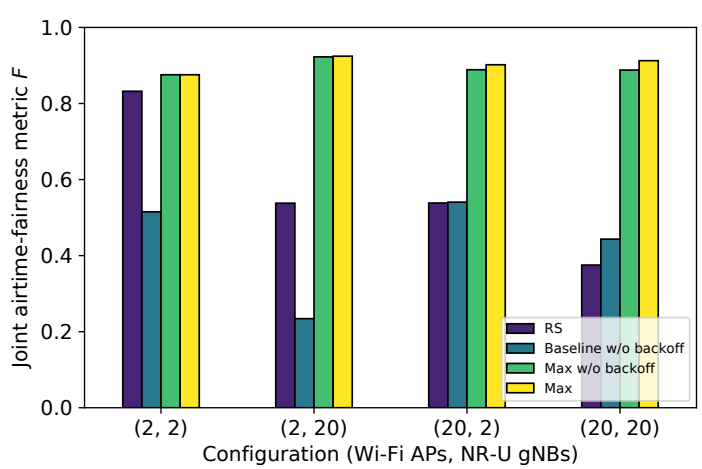

Figure 7: Simulation results comparing the joint airtimefairness metric under available channel access approaches: (a) reservation signal (NR-U uses RSs prior to transmission), (b) gap (gNBs do not use RSs, they are desynchronized and backoff is disabled), (c) Wi-Fi CW is optimized (NR-U uses gap settings), (d) both Wi-Fi and NR-U CWs are optimized.

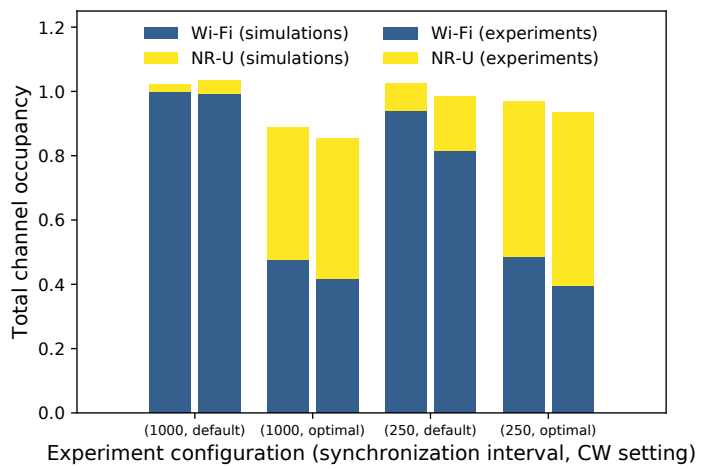

Figure 8: Comparison of simulation and testbed results.

- gap - gNBs operate under Observations 1 and 2 (desynchronized, backoff disabled) and wait a gap period prior to transmission,

- gap with optimal Wi-Fi CW - gNBs use gap settings but the Wi-Fi CW is optimized for equal channel access,

- gap with optimal CWs - gNBs use gap settings and both Wi-Fi and NR-U CWs are optimized.

Figure 7 presents the simulation results for four different configurations of competing APs and gNBs. The reservation signal approach is the baseline (it also corresponds to the case where $\mathrm{Wi}-\mathrm{Fi}$ is contending with other Wi-Fi nodes). If gNBs switch from RS to a gap-based approach, the overall network performance is worse (and would be even worse if these nodes were synchronized and used backoff). Fortunately, equalizing the channel access delay by optimizing Wi-Fi's CW settings is enough to considerably improve performance and surpass even the baseline. Finally, optimizing both Wi-Fi's and NR-U's CW settings leads to an additional improvement.

In general, optimizing CW leads to values higher than the standard CWmin and this may lead to unwanted increased delay. We 
measured the channel access delay, defined as the time between the instant a node begins contending for channel access (for a given Wi-Fi data frame or NR-U subframes) and the instant it starts the transmission (or the frame is dropped). Simulations confirmed (results not shown due to the lack of space) that despite the higher $\mathrm{CW}$ values, the channel access delay was always lower than the baseline, on account of the reduced collision rate.

OBSERVATION 4. The airtime fairness solution arrived at by $C W$ tuning is better in terms of network performance for both Wi-Fi and $N R-U$ than in the case of NR-U using reservation signals.

We confirmed this observation in our experimental testbed which consists of Wireless open-Access Research Platform (WARP) software defined radios (SDRs). The WARPs are based on field-programmable gate array (FPGA) circuits to exploit both PHY and MAC layer programmability. For Wi-Fi we used an existing implementation and for NR-U - we implemented its PHY and MAC operation as shown in [13]. At the PHY layer, the Wi-Fi nodes used appropriate transmission rate and frame length settings to achieve a data transmission duration of $2 \mathrm{~ms}$ whereas for NR-U we were able to set the transmission duration directly. We used five WARPs, placed in near proximity, to set up the over-the-air validation scenario: two Wi-Fi links composed of a receiver and two transmitters, and two NR-U transmitters. All parameters were configured so as to replicate the simulation scenario. Figure 8 presents an airtime metric for both Wi-Fi and NR-U for the two extreme synchronization intervals available for NR-U in the $5 \mathrm{GHz}$ band (250 and $1000 \mu$ s) and for two CW settings (default and optimal). The airtime metric is the normalized channel occupancy time, defined as all the time nodes using a given channel access method are transmitting (regardless of whether the transmission ended successfully or in a collision) normalized to the total time. Therefore, in this figure, for both simulations and experiments, the metrics includes all transmission attempts, i.e., both successful and collided transmissions, which means that it can exceed a value of one. Recall that for NR-U collisions are handled in the licensed bands and it is not always possible to distinguish collisions from successful transmissions while analyzing the unlicensed channel. The presented results both validate the simulation model and show that under optimal $\mathrm{CW}$ values the airtime fairness is improved.

Despite the positive conclusion of Observation 4, there is no onesize-fits-all CW setting and simulations for finding the optimal CW setting for given conditions are time consuming. In the next section, we demonstrate how we can derive the CW/NR-U parameters, resulting with satisfactory airtime-fairness, in practice.

\section{REGRESSION MODEL FOR WI-FI/NR-U CW PREDICTIONS}

We have developed a multi-input multi-output regression model for coexisting Wi-Fi and NR-U nodes to predict CW settings resulting in fair channel access. We used LSTM (Long Short-Term Memory) networks-based machine learning, which was successfully applied in the past, e.g., for interference prediction in IoT networks [24]. We have also verified that this regression type gives better results than the multi-layer perceptron regressor (not shown in the paper due to the lack of space).

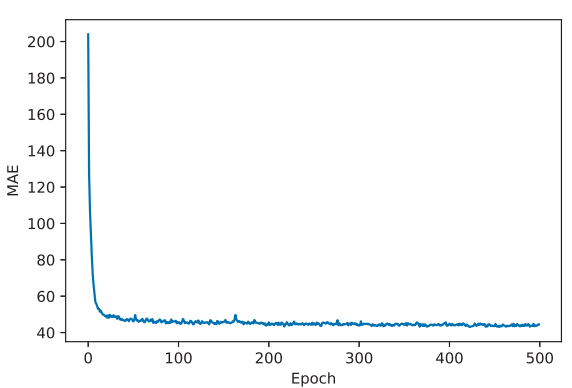

Figure 9: MAE loss function during training.

\subsection{Model Definition and Training}

First, we used factorial optimization to find the MAC parameters that impact the quality of $\mathrm{Wi}-\mathrm{Fi} / \mathrm{NR}-\mathrm{U}$ coexistence. As a result the following input parameters were considered: number of Wi-Fi nodes $\left(N_{W}\right)$, number of NR-U nodes $\left(N_{N}\right)^{5}$, synchronization period length $(\Delta)$, and transmission duration $(L)$. Additionally, $C W_{W}$ and $C W_{N}$ were selected as output parameters. Then, we gathered 40,800 samples from the simulator described in Section 3.3 for the following settings: $N_{W}, N_{N} \in\{1,5,10,15,20\}, C W_{W} \in$ $\{31,63, \ldots, 511,575, \ldots, 1023\}$ (24 settings in total), $C W_{N} \in\{0,3,7$, $11, \ldots, 63\}$ (17 settings in total), $\Delta \in\{250,1000\}[\mu \mathrm{s}], L \in\{6$, $2.1\}$ [ms]. Finally, we pruned the training data to up to 20 best samples for each training configuration, under the assumption of $F \geq 0.88$ (for several points this was the best $F$ we found ${ }^{6}$ ). This way we obtained 1,920 final samples for LSTM model training.

Next, we implemented a sequential model composed of an LSTM input layer with a Rectified Linear Unit (ReLU) activation function, a dense output layer with a linear activation function, and an Adam optimizer with a mean absolute error (MAE) loss function. The model was implemented using Python and Keras.Then, using grid search, we found the following hyper-parameters of the model: epochs $=500$, batch size $=256$, units $=1024$, dropout rate $=0.5$, which allowed to successfully fit the model to the training data. Figure 9 shows the changes of MAE as a function of the epoch number. The results stabilized at an MAE of about 50, which confirms that the selected hyper-parameters are correct.

\subsection{Testing the Regression Model}

We tested the implemented regression model using the 24 configurations given in Table 2. To validate the predicted results, we first gathered 9,792 samples from the simulator described in Section 3.3 for the following settings: $N_{W} \in\{2,12,24\}, N_{N} \in\{2,3\}, C W_{W} \in$ $\{31,63, \ldots, 511,575, \ldots, 1023\}$ (24 settings in total), $C W_{N} \in\{0,3,7$, $11, \ldots, 63\}$ (17 settings in total), $\Delta \in\{250,1000\} \mu \mathrm{s}, L_{W}, L_{N} \in$ $\{6,2.1\} \mathrm{ms}$. Then, we pruned the gathered test data, similarly as for the training data. We obtained 463 samples with $F \geq 0.88$, which served as a comparison for the predicted $\mathrm{CW}$ values.

The CW values predicted by the regression model are presented in Table 3. It can be noticed that for the increasing length of $\Delta$-the $C W_{W}$ size increases and $C W_{N}$ decreases. This is a result of the

\footnotetext{
${ }^{5}$ In a practical setting, the values of $N_{W}$ and $N_{N}$ can be estimated, e.g., using machine learning [35].

${ }^{6}$ For legacy Wi-Fi/NR-U operation $F$ was much lower, as shown in Section 5.3.
} 
Table 2: Settings of the test configurations.

\begin{tabular}{|c|rrrr|r|rrrr|}
\hline No. & $N_{W}$ & $N_{N}$ & $\Delta$ & $L$ & No. & $N_{W}$ & $N_{N}$ & $\Delta$ & $L$ \\
\hline 1 & 2 & 2 & 250 & 2.1 & 13 & 2 & 2 & 250 & 6 \\
2 & 2 & 2 & 1000 & 2.1 & 14 & 2 & 2 & 1000 & 6 \\
3 & 2 & 3 & 250 & 2.1 & 15 & 2 & 3 & 250 & 6 \\
4 & 2 & 3 & 1000 & 2.1 & 16 & 2 & 3 & 1000 & 6 \\
5 & 12 & 2 & 250 & 2.1 & 17 & 12 & 2 & 250 & 6 \\
6 & 12 & 2 & 1000 & 2.1 & 18 & 12 & 2 & 1000 & 6 \\
7 & 12 & 3 & 250 & 2.1 & 19 & 12 & 3 & 250 & 6 \\
8 & 12 & 3 & 1000 & 2.1 & 20 & 12 & 3 & 1000 & 6 \\
9 & 24 & 2 & 250 & 2.1 & 21 & 24 & 2 & 250 & 6 \\
10 & 24 & 2 & 1000 & 2.1 & 22 & 24 & 2 & 1000 & 6 \\
11 & 24 & 3 & 250 & 2.1 & 23 & 24 & 3 & 250 & 6 \\
12 & 24 & 3 & 1000 & 2.1 & 24 & 24 & 3 & 1000 & 6 \\
\hline
\end{tabular}

impact of $\Delta$ on the probability of NR-U transmission. Additionally, the size of $C W_{N}$ is always smaller than the size of $C W_{W}$, because the gap mechanism serves as a partial backoff, as discussed in Section 4.

Table 3: Optimal CW values found using different methods: predicted by the regression model, tuned using the analytical model of Section 3.2, found as having highest airtimefairness $F$ using the simulator of Section 3.3. The first column represents the test configuration number.

\begin{tabular}{|r|rr|rr|rr|}
\cline { 2 - 7 } \multicolumn{1}{c|}{} & \multicolumn{2}{|l|}{ Predicted } & Tuned & & \multicolumn{2}{|c|}{ Simulated } \\
\hline No. & $C W_{W}$ & $C W_{N}$ & $C W_{W}$ & $C W_{N}$ & $C W_{W}$ & $C W_{N}$ \\
\hline 1 & 93 & 31 & 77 & 14 & 63 & 7 \\
2 & 231 & 6 & 179 & 1 & 191 & 3 \\
3 & 103 & 31 & 105 & 23 & 63 & 0 \\
4 & 247 & 5 & 189 & 1 & 191 & 0 \\
5 & 204 & 43 & 244 & 64 & 223 & 55 \\
6 & 312 & 15 & 303 & 11 & 255 & 3 \\
7 & 221 & 41 & 257 & 64 & 255 & 55 \\
8 & 323 & 15 & 326 & 13 & 255 & 3 \\
9 & 317 & 52 & 324 & 64 & 351 & 63 \\
10 & 511 & 27 & 569 & 38 & 575 & 31 \\
11 & 373 & 49 & 336 & 64 & 383 & 63 \\
12 & 528 & 27 & 767 & 63 & 639 & 39 \\
13 & 83 & 14 & 146 & 63 & 127 & 31 \\
14 & 221 & 9 & 179 & 1 & 159 & 0 \\
15 & 91 & 15 & 166 & 64 & 127 & 27 \\
16 & 237 & 9 & 189 & 1 & 191 & 3 \\
17 & 174 & 25 & 250 & 64 & 255 & 63 \\
18 & 388 & 27 & 498 & 50 & 479 & 39 \\
19 & 183 & 26 & 259 & 64 & 287 & 63 \\
20 & 410 & 28 & 544 & 56 & 511 & 43 \\
21 & 275 & 36 & 328 & 64 & 383 & 63 \\
22 & 684 & 52 & 707 & 64 & 703 & 55 \\
23 & 293 & 35 & 336 & 64 & 383 & 63 \\
24 & 702 & 52 & 831 & 64 & 767 & 63 \\
\hline
\end{tabular}

Additionally, in Figure 10 for each test configuration we compare three metrics (per-node throughput, normalized airtime $A$, and joint airtime-fairness $F$ ) obtained with (i) the predicted $\mathrm{CW}$ settings (given in Table 3 as "Predicted"), (ii) CW settings tuned by the analytical model (given in Table 3 as "Tuned"), and (iii) the best test CW settings found by our simulation campaign (given in Table 3 as "Simulated") which resulted with the maximum values for each of the test scenarios. All results are very close each other. However, in some cases the predicted CW settings result in slightly better performance than the simulated/tuned ones. This is because the simulation campaign did not cover all possible CW sizes and for some test scenarios it provided satisfactory, though sub-optimal results. Additionally, the model measured fairness using Jain's fairness index calculated over the per-technology average per-node

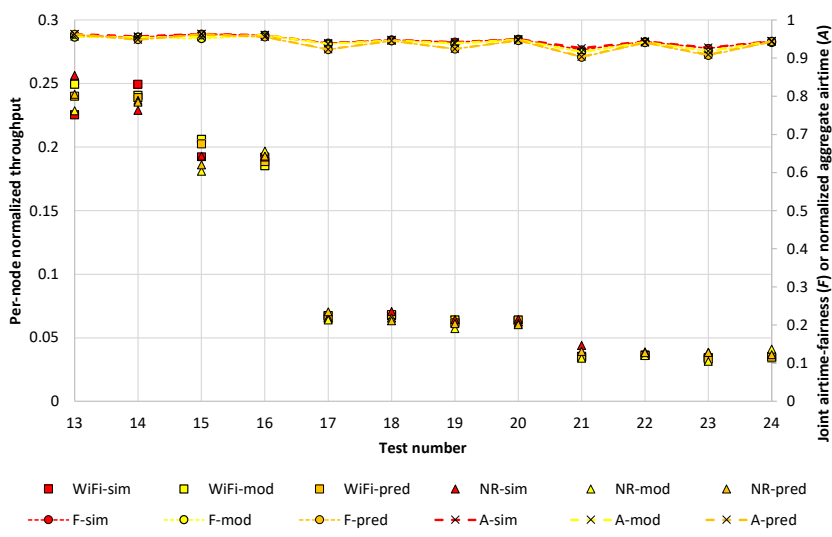

Figure 10: Comparison of results for CWs predicted by the proposed regression model (pred), tuned using the analytical model (mod), and with the maximum test results (sim) for $L=6 \mathrm{~ms}$.

normalized airtime, and not the normalized airtime of each node used by the simulator. For clarity of presentation we only show test scenarios 13-24; for other scenarios the results were equally good.

\subsection{Comparison with Legacy Operation}

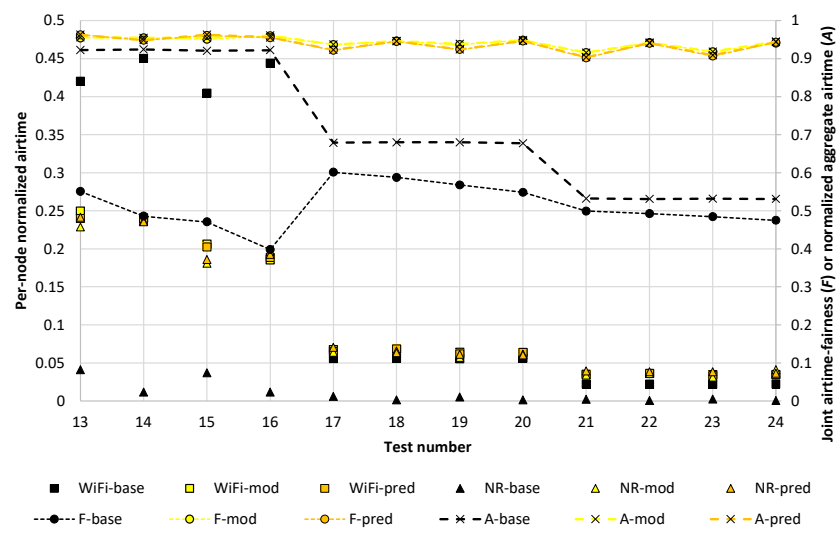

Figure 11: Comparison of results for CWs predicted by the proposed regression model ( pred), tuned using the analytical model $(\bmod )$, and with legacy operation (base) for $L=6 \mathrm{~ms}$.

In Figure 11, we compare three metrics (per-node throughput, normalized airtime $A$, and joint airtime-fairness $F$ ) obtained using the predicted (pred) and tuned (mod) CW settings with the legacy operation of both Wi-Fi and NR-U (base), i.e., when $C_{W}^{\min }=$ $C_{N}^{\min }=15$ and $C_{W}^{\max }=C_{N}^{\max }=63$. The results confirm the findings from Section 3.3. The proposed approach greatly improves airtimefairness in channel access for coexisting Wi-Fi and NR-U networks of different size, i.e., the NR-U nodes are no longer outperformed by the Wi-Fi nodes and the $A$ values are higher than for legacy operation. The highest gain in terms of the $A$ is observed for a large disproportion in the number of coexisting Wi-Fi and NR-U nodes. 
For all configurations $F \approx 0.9$ for the proposed approach. Again, for clarity of presentation we only show test scenarios 13-24. For other scenarios the results were equally good.

\section{CONCLUSIONS}

Inspired by the growing interest in assuring coexistence fairness between random (e.g., Wi-Fi) and scheduled (e.g., LTE-LAA, NR-U) wireless networks implementing LBT channel access, in this paper we proposed a re-evaluation of the reservation-less gap mechanism. We showed that by treating gap periods as a partial backoff, it is possible to preserve fairness in coexistence and to avoid the necessity of implementing reservation signals in 5G networks.

Having developed both analytical and simulation models, we validated them in a testbed to show the advantages of our proposed approach. Additionally, we have shown how the analytical model could be used to tune the CW settings for Wi-Fi and NR-U. Furthermore, we developed an LSTM-based regression model to show that it is possible to derive $\mathrm{Wi}-\mathrm{Fi} / \mathrm{NR}-\mathrm{U} \mathrm{CW}$ values resulting in satisfactory joint fairness-airtime coexistence in practice.

The proposed approach is especially useful for long synchronization periods (i.e., lengths allowed for NR-U in the $5 \mathrm{GHz}$ band), since for short synchronization periods (symbol-based scheduling) the behaviour Wi-Fi and NR-U converges [13]. However, it remains to be seen whether such symbol-based scheduling can be implemented in practical NR-U deployments. As future work, we plan to consider uplink transmissions and partially overlapping networks (with hidden nodes) as well as online learning frameworks [9].

\section{ACKNOWLEDGMENTS}

This work was carried out as part of a project financed by the Polish National Science Centre (DEC-2018/30/M/ST7/00351).

\section{REFERENCES}

[1] IEEE Standard for Information technology-Telecommunications and information exchange between systems Local and metropolitan area networks-Specific requirements - Part 11: Wireless LAN Medium Access Control (MAC) and Physical Layer (PHY) Specifications. IEEE Std 802.11-2016, pages 1-3534, Dec 2016.

[2] $5 \mathrm{GHz}$ RLAN; Harmonised Standard covering the essential requirements of article 3.2 of Directive 2014/53/EU, 2017.

[3] 3GPP. LTE; Physical layer procedures for shared spectrum channel access (Release 16). Technical Specification (TS) 37.213, 3rd Generation Partnership Project (3GPP), 12 2020. Version 16.4.0.

[4] R. Ali, B. Kim, S. W. Kim, H. Kim, and F. Ishmanov. (ReLBT): A Reinforcement learning-enabled listen before talk mechanism for LTE-LAA and Wi-Fi coexistence in IoT. Computer Communications, 150:498-505, 2020.

[5] Z. Ali, L. Giupponi, J. Mangues-Bafalluy, and B. Bojovic. Machine learning based scheme for contention window size adaptation in LTE-LAA. In 2017 IEEE 28th Annual International Symposium on Personal, Indoor, and Mobile Radio Communications (PIMRC), pages 1-7, 2017.

[6] G. Bianchi et al. Performance analysis of the IEEE 802.11 distributed coordination function. IEEE fournal on Selected Areas in Communications, 18(3):535-547, 2000

[7] U. Challita and M. K. Marina. Holistic small cell traffic balancing across licensed and unlicensed bands. In 19th ACM International Conference on Modeling, Analysis and Simulation of Wireless and Mobile Systems, MSWiM '16, page 166-175, 2016.

[8] M. Cierny, T. Nihtila, T. Huovinen, M. Kuusela, F. Chernogorov, K. Hooli, and A. Toskala. Fairness vs. Performance in Rel-13 LTE Licensed Assisted Access. IEEE Communications Magazine, 55(12):133-139, 2017.

[9] P. Gawłowicz and A. Zubow. ns-3 meets OpenAI Gym: The Playground for Machine Learning in Networking Research. In 22nd ACM International Conference on Modelling, Analysis and Simulation of Wireless and Mobile Systems (MSWiM 2019), Miami Beach, FL, Nov. 2019. ACM. doi: 10.1145/3345768.3355908.

[10] M. Han, S. Khairy, L. X. Cai, Y. Cheng, and R. Zhang. Reinforcement Learning for Efficient and Fair Coexistence Between LTE-LAA and Wi-Fi. IEEE Transactions on Vehicular Technology, 69(8):8764-8776, 2020.
[11] M. Hirzallah, M. Krunz, and Y. Xiao. Harmonious cross-technology coexistence with heterogeneous traffic in unlicensed bands: Analysis and approximations. IEEE Tr. on Cognitive Communications and Networking, 5(3):690-701, 2019.

[12] C.-Y. Huang, H.-Y. Chen, C.-H. Huang, S.-T. Sheu, T.-W. Chiang, T.-L. Cheng, and C.-C. Chang. Listen Before Receive (LBR) Assisted Network Access in LAA and WiFi Heterogeneous Networks. IEEE Access, 9:43845-43861, 2021.

[13] K. Kosek-Szott, A. Lo Valvo, S. Szott, P. Gallo, and I. Tinnirello. Downlink channel access performance of NR-U: Impact of numerology and mini-slots on coexistence with Wi-Fi in the $5 \mathrm{GHz}$ band. Computer Networks, 195:108188, 2021. ISSN 1389-1286.

[14] P. Kutsevol, V. Loginov, E. Khorov, and A. Lyakhov. New Collision Detection Method for Fair LTE-LAA and Wi-Fi Coexistence. In 2019 IEEE 30th Annual International Symposium on Personal, Indoor and Mobile Radio Communications (PIMRC), pages 1-6, 2019.

[15] S. Lagen and L. Giupponi. Listen before receive for coexistence in unlicensed mmWave bands. In 2018 IEEE Wireless Communications and Networking Conference (WCNC), pages 1-6, 2018. doi: 10.1109/WCNC.2018.8377293.

[16] V. Loginov, E. Khorov, A. Lyakhov, and I. Akyildiz. CR-LBT: Listen-before-Talk with Collision Resolution for 5G NR-U Networks. IEEE Transactions on Mobile Computing, pages 1-1, 2021. doi: 10.1109/TMC.2021.3055028.

[17] V. A. Loginov, A. I. Lyakhov, and E. M. Khorov. Coexistence of Wi-Fi and LTE-LAA Networks: Open Issues. Journal of Communications Technology and Electronics, 63(12):1530-1537, 2018. ISSN 10642269. doi: 10.1134/S1064226918120148.

[18] M. Mehrnoush, V. Sathya, S. Roy, and M. Ghosh. Analytical Modeling of Wi-Fi and LTE-LAA Coexistence: Throughput and Impact of Energy Detection Threshold. IEEE/ACM Transactions on Networking, 26(4):1990-2003, 2018. ISSN 10636692.

[19] S. Mosleh, Y. Ma, J. D. Rezac, and J. B. Coder. A Novel Machine Learning Approach to Estimating KPI and PoC for LTE-LAA-based Spectrum Sharing. In 2020 IEEE International Conference on Communications Workshops (ICC Workshops), pages 1-6. IEEE, 2020.

[20] A. Mukherjee, J.-F. Cheng, S. Falahati, H. Koorapaty, R. Karaki, L. Falconetti, D. Larsson, et al. Licensed-assisted access LTE: coexistence with IEEE 802.11 and the evolution toward 5G. IEEE Communications Magazine, 54(6):50-57, 2016.

[21] A. Myles. Agenda for IEEE 802.11 Coexistence SC meeting in Hawaii in November 2019". Technical Report IEEE 802.11-19/1763r6, IEEE 802.11, 2019.

[22] G. Naik, Jung-Min, Park, J. Ashdown, and W. Lehr. Next Generation Wi-Fi and 5G NR-U in the $6 \mathrm{GHz}$ Bands: Opportunities \& Challenges. 2020. URL http://arxiv.org/abs/2006.16534.

[23] P. Nikolich. Liaison Statement to 3GPP from IEEE 802 LMSC. IEEE 802.19-16/0037r9, IEEE 802, 2016. URL https://mentor.ieee.org/802.19/dcn/16/ 19-16-0037-09-0000-laa-comments.pdf.

[24] A. Nikoukar, Y. Shah, A. Memariani, M. Güneş, and B. Dezfouli. Predictive Interference Management for Wireless Channels in the Internet of Things. In 2020 IEEE 31st Annual International Symposium on Personal, Indoor and Mobile Radio Communications, pages 1-7. IEEE, 2020.

[25] N. Patriciello, S. Lagen, B. Bojovic, and L. Giupponi. NR-U and IEEE 802.11 Technologies Coexistence in Unlicensed mmWave Spectrum: Models and Evaluation. IEEE Access, 8:71254-71271, 2020. ISSN 2169-3536.

[26] E. Pei and J. Jiang. Performance analysis of licensed-assisted access to unlicensed spectrum in LTE Release 13. IEEE Transactions on Vehicular Technology, 68(2): 1446-1458, 2019. ISSN 00189545. doi: 10.1109/TVT.2018.2886332.

[27] S. Saadat, W. Ejaz, S. Hassan, I. Bari, and T. Hussain. Enhanced network sensitive access control scheme for LTE-LAA/WiFi coexistence: Modeling and performance analysis. Computer Communications, 172:45-53, 2021. ISSN 0140-3664.

[28] R. Saleem, S. A. Alvi, and S. Durrani. Performance-Fairness Trade-off for Wi-Fi and LTE-LAA Coexistence. IEEE Access, 2021.

[29] J. Tan, L. Zhang, Y.-C. Liang, and D. Niyato. Intelligent sharing for LTE and WiFi systems in unlicensed bands: A deep reinforcement learning approach. IEEE Transactions on Communications, 68(5):2793-2808, 2020.

[30] I. Tinnirello and G. Bianchi. Rethinking the IEEE 802.11e EDCA performance modeling methodology. IEEE/ACM transactions on networking, 18(2):540-553, 2009.

[31] I. Tinnirello, P. Gallo, S. Szott, and K. Kosek-Szott. Impact of LTE's Periodic Interference on Heterogeneous Wi-Fi Transmissions. IEEE Communications Letters, 23(2):342-345, 2018.

[32] J. Verboom and S. Kim. Stochastic Analysis on Downlink Performance of Coexistence between WiGig and NR-U in $60 \mathrm{GHz}$ Band. arXiv preprint arXiv:2003.01570, 2020.

[33] A. M. Voicu, L. Simić, and M. Petrova. Survey of Spectrum Sharing for InterTechnology Coexistence. IEEE Communications Surveys \& Tutorials, 21(2):11121144, 2019. doi: 10.1109/COMST.2018.2882308.

[34] J. Wszołek, S. Ludyga, W. Anzel, and S. Szott. Revisiting LTE LAA: Channel Access, QoS, and Coexistence with WiFi. IEEE Communications Magazine, 59(2): 91-97, 2021. doi: 10.1109/MCOM.001.2000595.

[35] B. Yang, X. Cao, Z. Han, and L. Qian. A machine learning enabled MAC framework for heterogeneous Internet-of-Things networks. IEEE Transactions on Wireless Communications, 18(7):3697-3712, 2019. 